\title{
OPTICAL INVESTIGATIONS OF PHASE TRANSITIONS IN $\left(\mathrm{NH}_{4}\right)_{2} \mathrm{SbF}_{5}$ CRYSTAL
}

\author{
B. Andriyevsky ${ }^{a}$, Z. Czapla ${ }^{b}$ AND V. StadnyK ${ }^{a}$ \\ ${ }^{a}$ Lviv State I. Franko University, Kyryla and Mefodiya 8, 290005 Lviv, Ukraine \\ ${ }^{b}$ Institute of Experimental Physics, University of Wroclaw \\ Pl. M. Borna 9, 50-204 Wrocław, Poland
}

(Received March 29, 1994; revised version September 6, 1994)

\begin{abstract}
Results of optical thickness and refractive indices measurements for $\left(\mathrm{NH}_{4}\right)_{2} \mathrm{SbF}_{5}$ crystal in the temperature range $100-310 \mathrm{~K}$ are presented. The measurements were done with interference method using $\mathrm{He}-\mathrm{Ne}$ laser $(\lambda=632.8 \mathrm{~nm})$. At $T_{\mathrm{c} 1}=292 \mathrm{~K}$ and $T_{\mathrm{c} 2}=168 \mathrm{~K}$ two continuous phase transitions with critical indices $\beta_{1}=0.38$ and $\beta_{2} \leq 0.57$ were observed. Additional anomaly of temperature changes of interference order with maximum at $T_{c 3} \approx 132 \mathrm{~K}$ were found. This anomaly resembles a diffused transition. Temperature-averaged values of derivatives of refractive indices $\mathrm{d} n_{\mathrm{g}} / \mathrm{d} T=$ $-3.3 \times 10^{-5} \mathrm{~K}$ and $\mathrm{d} n_{\mathrm{m}} / \mathrm{d} T=-2.9 \times 10^{-5} \mathrm{~K}^{-1}$ evidenced a decrease in optical anisotropy (birefringence) along the $b$-axis on heating run.
\end{abstract}

PACS numbers: $64.70 . \mathrm{Kb}, 78.20 . \mathrm{Fm}$

\section{Introduction}

Crystal of $\left(\mathrm{NH}_{4}\right)_{2} \mathrm{SbF}_{5}$ ammonium pentafluoroantimonate (APFA) belongs to the big family of compounds with chemical formula $\mathrm{M}_{2} \mathrm{SbF}_{5}$, where $\mathrm{M}=\mathrm{Na}$, $\mathrm{K}, \mathrm{Rb}, \mathrm{Cs}, \mathrm{NH}_{4}$. Successive phase transitions were reported in $\left(\mathrm{NH}_{4}\right)_{2} \mathrm{SbF}_{5}$ crystal [1-8]. However, there are discrepancies related to the number of the phase transitions in this compound. In a paper [6] the proposed phase transitions and symmetry of particular phases are recollected. In the temperature range $110-330 \mathrm{~K}$ four phase transitions at $138,165,257$ and $294 \mathrm{~K}$ are reported. At $298 \mathrm{~K}$ (phase I) the crystal belongs to orthorhombic system with the space group $\mathrm{Cmcm}$ [7]. In the temperature range $138-294 \mathrm{~K}$ monoclinic symmetry (space group $C 2 / c$ ) is proposed. In the papers [1-3] a transition to superionic phase at $257 \mathrm{~K}$ is implied. However, nuclear quadrupole resonance (NQR) measurements [5] gave evidences for two phase transitions at $292 \mathrm{~K}$ and $169 \mathrm{~K}$ only. Additionally the unusual behaviour of resonance frequency below $169 \mathrm{~K}$ is observed. Temperature dependences of lattice parameters [6] confirmed two phase transitions and proved the 
change of symmetry from orthorhombic to monoclinic at about $294 \mathrm{~K}$. Optical observations [8] showed the ferroelastic character of the phase transition at $292 \mathrm{~K}$. Dielectric measurements showed the change of slope of $\varepsilon^{\prime}$ at $169 \mathrm{~K}$ and its decrease in the temperature range $169-120 \mathrm{~K}$. The aim of the presented paper is to obtain additional information related to the phase transitions sequence and description of phase transitions character.

\section{Experimental}

APFA single crystals of good quality were grown from water solution containing stoichiometric quantities of $\mathrm{NH}_{4} \mathrm{~F}$ and $\mathrm{SbF}_{3}$ with a small excess of $\mathrm{HF}$ by slow evaporation at constant temperature of $301 \mathrm{~K}$. The crystal exhibits a very distinct cleavage plane (010) perpendicular to the $b$-axis. In our work the $b$-cut samples were used in optical investigations. Optical observations of APFA samples with polarization microscope at room temperature showed that the crystal is negative biaxial one. The least $n_{\mathrm{p}}$-halfaxis of optical indicatrix is identical to the crystallophysical $b$-axis, the greatest $n_{\mathrm{g}}$ - to the $a$-axis and the middle $n_{\mathrm{m}}$ - to the $c$-axis $\left(n_{\mathrm{g}}>n_{\mathrm{m}}>n_{\mathrm{p}}\right)$. The parallel surfaces of the samples (necessary in interference method) were obtained by cleavage of the crystal. The optical interference method used in measurements is presented schematically in Fig. 1.

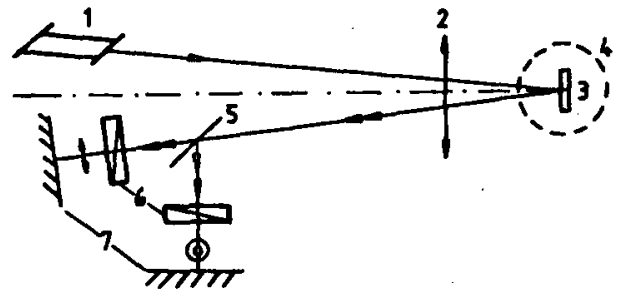

Fig. 1. Scheme of optical experimental setup: $1-\mathrm{He}-\mathrm{Ne}$ laser $(\lambda=632.8 \mathrm{~nm})$, 2 - lens, 3 - sample, 4 - cryostat, 5 - semitransparent mirror, 6 - polarizers, 7 - screens.

The laser beam was focussed by means of lens (2) on the sample (3) which was placed in cryostat (4). The incident angle of laser beam was $\alpha=3^{\circ}$. Therefore, an influence of optical anisotropy for the optical thickness of APFA in the plane of incident beam was negligible. As a result of interference of two beams reflected from front and back surfaces of the sample one observes two interference pictures on the screens (7). The direction of linear polarization of laser beam corresponded to the bisectrix of right angle between $n_{\mathrm{g}^{-}}$and $n_{\mathrm{m}}$-axes. Two polarizers (6) oriented along $n_{\mathrm{g}^{-}}$and $n_{\mathrm{m}}$-axes allowed to obtain two interference pictures at every temperature point. Thus, in this experiment temperature dependences of two double optical thicknesses changes $\delta \Delta_{\mathrm{g}}=2 \delta\left(n_{\mathrm{g}} \cdot l\right)$ and $\delta \Delta_{\mathrm{m}}=2 \delta\left(n_{\mathrm{m}} \cdot l\right)$ of APFA crystal were measured simultaneously. Here, $l$ is the geometric thickness of a sample. The diameter $0.5-0.6 \mathrm{~mm}$ of laser beam did not provide the condition of monodomain investigation taking into account small width $(15-20 \mu \mathrm{m})$ of 
APFA domains [8]. But temperature dependent distinct interference effect in the whole range $100-310 \mathrm{~K}$ evidences small birefringence variation due to ferroelastic domain structure with respect to acting values of refractive indices. Therefore, one can say that the domain structure does not influence essentially the temperature dependence of optical thickness. As well known, as effects of temperature hysteresis for second order phase transitions are small, the presented investigations of APFA were performed at continuous increase in temperature with the rate of $\approx 1 \mathrm{~K} / \mathrm{min}$. To obtain relative temperature changes of $\delta \Delta / \Delta$, refractive index $n$ and geometric thickness of the sample were measured at room temperature with the relative accuracies $10^{-4}$ and $10^{-2}$, respectively. Refractive indices of APFA at room temperature were measured by the immersion and photographic Obreimov's methods [9]. Refractive indices at $T_{0}=295 \mathrm{~K}$ are equal to: $n_{\mathrm{g}}=1.5266, n_{\mathrm{m}}=1.5178$ and $n_{\mathrm{p}}=1.4703$. The temperature changes of double optical thickness $\delta \Delta$ were estimated with accuracy $\lambda / 4$. Using the values of $n \approx 1.5$ and $l \approx 1.5 \mathrm{~mm}$ the average relative error of determination of double optical thickness variations is equal to $7 \times 10^{-5}$. Using dependence of $\delta \Delta / \Delta=f(T)$ (obtained in this experiment) and temperature dependences of lattice constant [6] (obtained from Bond's method), the temperature changes of relative refractive indices $\delta n / n$ of APFA were obtained according to the formula

$$
\delta \Delta / \Delta=\delta n / n+\delta l / l \text {. }
$$

The used method is characterized by high temperature sensitivity. 350-380 interference extrema (number of points on plots) correspond to $110-310 \mathrm{~K}$ temperature range and $1.5 \mathrm{~mm}$ sample thickness. Temperature dependence of interference extrema changes was measured by thermocouple and photodiode with the help of $X-Y$ recorder.

\section{Results and conclusions}

Temperature dependences of wavelength-normalized temperature changes of double optical thickness $\delta \Delta_{\lambda}=2 \delta(n \cdot l) / \lambda$ of APFA for two polarizations of light $\boldsymbol{E} \| n_{\mathrm{g}}$ and $\boldsymbol{E} \| n_{\mathrm{m}}$ are shown in Fig. 2. The value $\delta \Delta_{\lambda}$ is equal to the change of interference maximum: $\Delta=k \lambda, \delta \Delta(T)=\delta k(T) \lambda, \delta \Delta_{\lambda}(T)=\delta \Delta(T) / \lambda=\delta k(T)$, where $\lambda$ is the wavelength of laser light. We shall omit $\lambda$-subindex in further text. These dependences showed similar temperature changes as the $b$-lattice constant (the $b$-axis is the direction of light propagation in this experiment). On heating both the $b$-lattice constant and $\delta \Delta$ increase without any discontinuity. At $T_{\mathrm{c} 1}=292 \mathrm{~K}$ and $T_{\mathrm{c} 2}=168 \mathrm{~K}$ the changes of both quantities are characteristic of continuous phase transition with a jump-like changes of $\mathrm{d}(\delta \Delta) / \mathrm{d} T$ (Fig. 2, Fig. 3). The temperature dependence of $\mathrm{d}(\delta \Delta) / \mathrm{d} T$ (curve 1 in Fig. 3 ) was calculated by numerical differentiation of the curve 1 in Fig. 2. Assuming that the induced by spontaneous deformation corresponding $\delta \Delta_{\text {s.d. }}$ value is related to order parameter with a formula

$$
\delta \Delta_{\text {s.d. }}^{\prime} \sim \eta^{2 \beta} \sim\left(T_{\mathrm{c}}-T\right)^{2 \beta},
$$

the critical indices for the phase transitions were obtained. The linear dependence $\lg (\delta \Delta)=2 \beta \lg \left(T_{\mathrm{c}}-T\right)$ is fulfilled satisfactorily in the $T_{\mathrm{c}}-\left(T_{\mathrm{c}}-4 \mathrm{~K}\right)$ temperature 


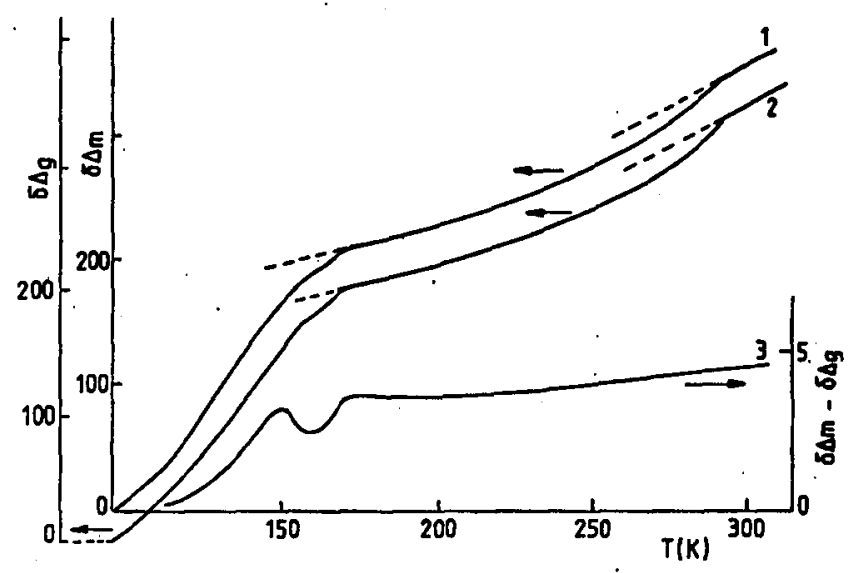

Fig. 2. Temperature dependences of normalized changes of double optical thickness $(\delta \Delta)$ for different light polarization $\left(1-E\left\|n_{\mathrm{m}}, 2-E\right\| n_{\mathrm{g}}\right)$ and their difference $\left(\delta \Delta_{\mathrm{m}}-\delta \Delta_{\mathrm{g}}\right)$ of $\left(\mathrm{NH}_{4}\right)_{2} \mathrm{SbF}_{5}$ crystal.

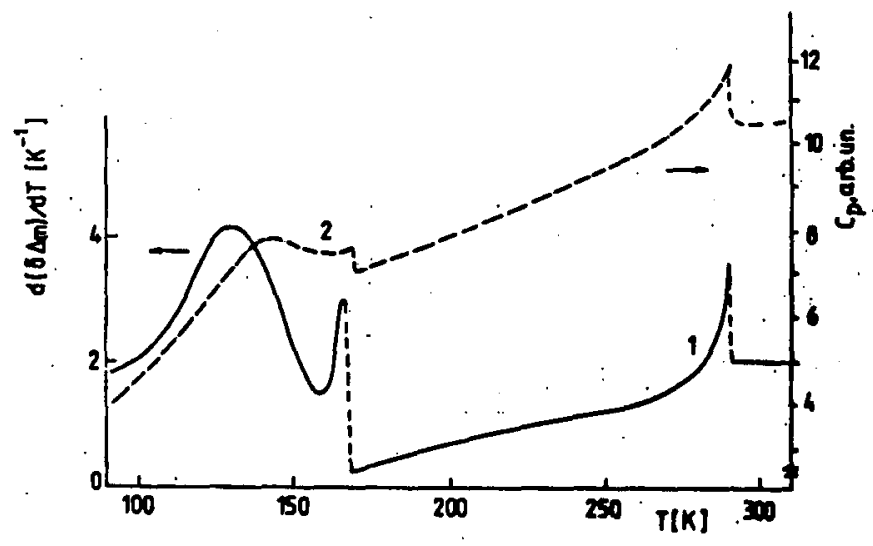

Fig. 3. Temperature dependences of normalized change of double optical thickness derivative $\mathrm{d}\left(\delta \Delta_{\mathrm{m}}\right) / \mathrm{d} T$ for light polarization $\boldsymbol{E} \| \boldsymbol{n}_{\mathrm{m}}(1)$ and heat capacity $C_{p}(2)$ of $\left(\mathrm{NH}_{4}\right)_{2} \mathrm{SbF}_{5}$ crystal.

region for both second order phase transitions (Fig. 4, Fig. 5). The calculation give values of $\beta_{1}=0.38 \pm 0.03$ and $\beta_{2}=0.57 \pm 0.04$ at $T_{\mathrm{c} 1}$ and $T_{\mathrm{c} 2}$, respectively. In the range $120-158 \mathrm{~K}$ large changes of $\delta \Delta$ are observed (Fig. 2). This behaviour is quite similar to that observed for the $b$-lattice parameter of elementary unit cell of APFA. Relatively strong temperature change of $\delta \Delta$ in the range 120-158 K resulting in pronounced but wide maximum of $\mathrm{d}(\delta \Delta) / \mathrm{d} T$ value (Fig. 3 ) resembles a diffused phase transition at $T_{\mathrm{c} 3} \approx 132 \mathrm{~K}$. This behaviour is in qualitative agreement with temperature dependences of $C_{p}$ [10] and dilatation [6] of APFA. The similarity of temperature dependences of $\mathrm{d}(\delta \Delta) / \mathrm{d} T$ and $C_{p}$ is shown in Fig. 3. Subtraction of $\delta \Delta$ contribution resulting from this diffused phase transition-like 


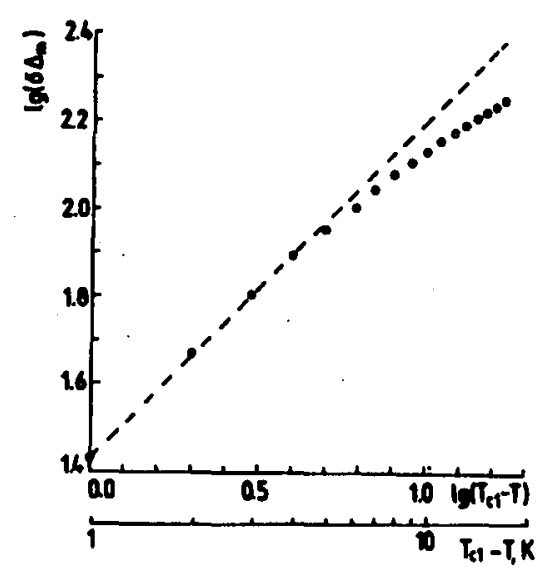

Fig. 4. Temperature dependence $\lg (\delta \Delta)=f\left[\lg \left(T_{\mathrm{c} 1}-T\right)\right]$ at the vicinity of phase transition at $T_{\mathrm{c} 1}=292 \mathrm{~K}$.

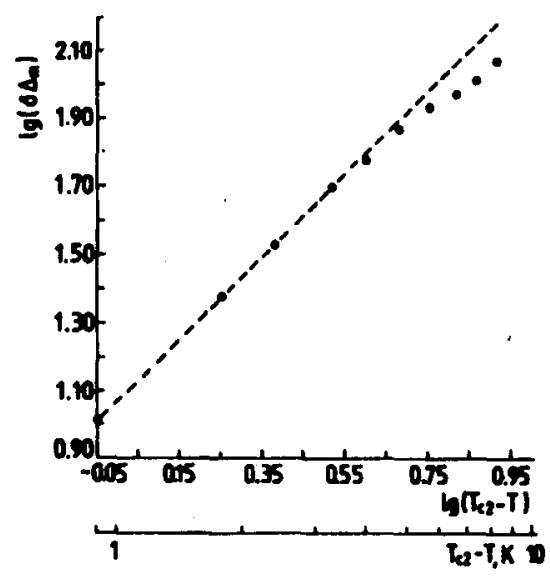

Fig. 5. Temperature dependence $\lg (\delta \Delta)=f\left[\lg \left(T_{\mathrm{c} 2}-T\right)\right]$ at the vicinity of phase transition at $T_{\mathrm{c} 2}=168 \mathrm{~K}$.

behaviour to the temperature changes of $\delta \Delta$ induced by the phase transition at $T_{\mathrm{c} 2}=168 \mathrm{~K}$ gives a decrease in the $\beta_{2}$ value to the value much better corresponding to continuous phase transition $(\beta<0.5)$ at this temperature $\left(\beta_{2}<0.57\right)$. Temperature dependences of double optical thickness changes $\delta \Delta$ (curves 1 and 2 in Fig. 2) do not allow to estimate temperature dependences of refractive indices of APFA. Temperature dependences of $\delta n / n$ were obtained according to the formula (1) using $\delta \Delta / \Delta$ data (this work) and $\delta l / l$ data [6]. On the ground of the $b$-lattice parameter temperature changes one can state that refractive indices along the $a$ and $c$-axes decrease $\delta n_{\mathrm{g}} / n_{\mathrm{g}}<0, \delta n_{\mathrm{m}} / n_{\mathrm{m}}<0$, and $\left|\delta n_{\mathrm{g}} / n_{\mathrm{g}}\right|>\left|\delta n_{\mathrm{m}} / n_{\mathrm{m}}\right|$. These relations and the values of refractive indices $n_{\mathrm{g}}>n_{\mathrm{m}}>n_{\mathrm{p}}$ give decrease in optical anisotropy, i.e. birefringence $\left(n_{\mathrm{g}}-n_{\mathrm{m}}\right)$ with increase in temperature. It is 


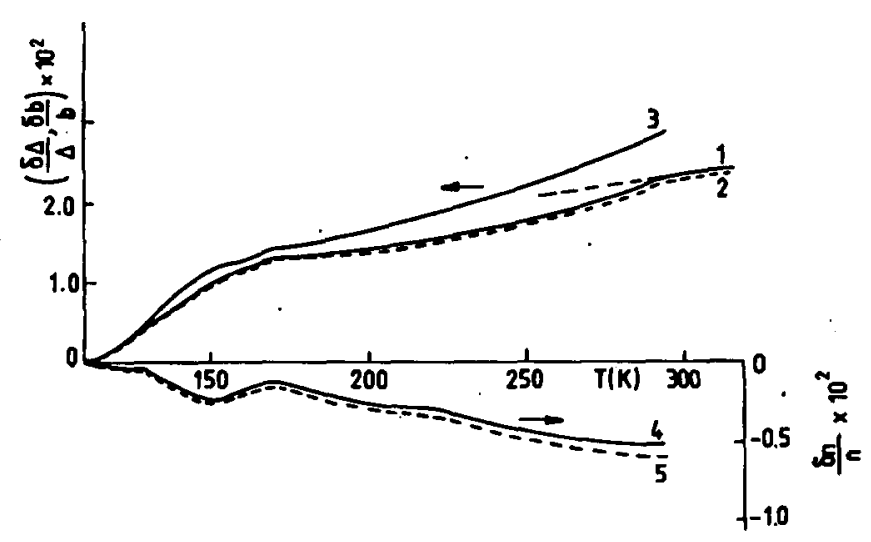

Fig. 6. Temperature dependences of relative changes of optical thickness $\delta \Delta / \Delta(1,2)$, $b$-lattice parameter, $\delta b / b(3)$ and refractive index $\delta n / n(4,5)$ of $\left(\mathrm{NH}_{4}\right)_{2} \mathrm{SbF}_{5}$ crystal for different light polarization $\left(1,4-E\left\|n_{\mathrm{m}}, 2,5-E\right\| n_{\mathrm{g}}\right)$.

seen in Fig. 6 that the greatest refractive index $n_{\mathrm{g}}$ decreases more rapidly than the middle one $n_{\mathrm{m}}$ at increase in temperature. Thus, one can expect the phase transition appearance to prototype tetragonal phase at higher temperature. According to data presented in Fig. 6 in the temperature range $110-310 \mathrm{~K}$ an average contribution $|\delta n / n|$ to the relationship (1) is equal to about $0.2|\delta l / l|$. The main contribution ( $80 \%)$ to the temperature changes of double optical thickness for both polarizations of light results from temperature changes of the $b$-parameter of the elementary unit cell according to the formula (1). On the contrary to the monotonical temperature increase in the $b$-lattice parameter $\mathrm{d} l / \mathrm{d} T>0$ in the range $110-295 \mathrm{~K}$ an anomalous increase in $\delta n / n$ is observed against a background of monotonically decreasing values of this quantity. Such behaviour corresponds to the temperature dependences of the $a$ - and $c$-lattice parameters of APFA [6]. The subtraction of temperature dependences ( 1 and 2 in Fig. 2) represents the temperature dependence of multiplication of birefringence $\left(n_{\mathrm{g}}-n_{\mathrm{m}}\right)$ and the $b$-lattice parameter. Anomalous behaviour of this dependence in the temperature range 150-170 K correlates well with a subtraction of the lattice parameters $(c-a)[6]$. The obtained results show that the anisotropy of the optical indicatrix of APFA in the plane of $n_{\mathrm{g}}-n_{\mathrm{m}}$ axes decreases over the temperature range 110-310 K.

To summarize the investigations of $\left(\mathrm{NH}_{4}\right)_{2} \mathrm{SbF}_{5}$ one can state:

1. using new modification of optical interference method the precise temperature dependences of double optical thickness were obtained along the $b$-axis in the temperature range $110-310 \mathrm{~K}(\lambda=632.8 \mathrm{~nm})$;

2. two continuous phase transitions are established at $T_{\mathrm{c} 1}=292 \mathrm{~K}$ and $T_{\mathrm{c} 2}=$ $168 \mathrm{~K}$ with critical indices $\beta_{1}=0.38$ and $\beta_{2} \leq 0.57$ respectively;

3. strong temperature changes of double optical thickness in the temperature range $120-158 \mathrm{~K}$ resemble the diffused phase transition at $T_{\mathrm{c} 3} \approx 132 \mathrm{~K}$.

This work was supported by the research project No. 201289101. 


\section{References}

[1] J.P. Grigas, R.L. Davydovitch, A. Petchelinnaite, V.V. Urbonavichius, Krist. Technik 14, 877 (1979).

[2] V.V. Urbonavichius, V.E. Shneider, J.P. Grigas, R.L. Davydovitch, Zh. Eksp. Teor. Fiz. 83, 275 (1982).

[3] L.M. Avkutskij, R.L. Davydovitch, L.A. Zemnukhova, P.S. Gordienko, V.V. Urbonavichius, J.P. Grigas, Phys. Status Solidi B 116, 483 (1983).

[4] Yu.Ya. Kharitonov, R.L. Davydovitch, V.I. Kostin, L.A. Zemnukhova, V.I. Sergienko, Zh. Strukt. Khim. 17, 1316 (1972).

[5] N. Nakamura, Z. Nat.forsch A 41, 243 (1986).

[6] A. Waśkowska, Z. Czapla, Cryst. Res. Technol. 24, 1259 (1989).

[7] R.R. Ryan, D.T. Cromer, Inorg. Chem. 11, 2322 (1972).

[8] Z. Czapla, S. Dacko, Ferroelectrics 140, 271 (1993).

[9] I.V. Obreimov, About Applications of Frehnel's Diffraction for the Physical and Technical Measurements, Acad. Sci. USSR, Moscow 1945, p. 81 (in Russian).

[10] A. Onodera, private communication. 